\title{
MÉTODO PILATES: UM ESTUDO DE SUAS \\ CARACTERÍSTICAS E A INFLUÊNCIA DA PRÁTICA NA POPULAÇÃO IDOSA*
}

\author{
Anderson Fagundes Ribeiro ${ }^{1}$ \\ Juliana Silva Guabiroba
}

\section{Introdução}

O Pilates é caraterizado como um tipo de treinamento físico e também mental, que entende o corpo e a mente como uma unidade, que busca desenvolver o organismo humano em sua totalidade para alcançar o melhor potencial em todos os aspectos. Esse método tem como base o fortalecimento da Resistência Muscular Localizada e da força, realizado a partir de um conjunto de exercícios de alongamento e fortalecimento, através de movimentos específicos e calmos que objetivam a união entre corpo, mente e espírito (APARICIO; PÉREZ,2005).

Esse método não é a prática de exercícios físicos praticados de forma casual, improvisada, mas, um completo conjunto de exercícios planejados que desenvolvem e aprimoram diversas capacidades físicas, além de diminuir o estresse, atuando como fator de promoção do bemestar tanto físico quanto mental (ISACOWITZ; CLIPPINGER, 2013).

Segundo Aparicio e Pérez (2005, p. 21-22):

O método Pilates é a completa coordenação de corpo, mente e espírito. Um sistema único de exercícios de alongamento e fortalecimento desenvolvido por Joseph H. Pilates há quase noventa anos, que trabalha em sequências de movimentos controlados e precisos, com aparelhos especializados criados pelo próprio Pilates, que oferecem um conjunto de resistências variáveis ao esforço muscular e atuam como os próprios músculos, combinando este trabalho com o de solo ou com colchonete.

\footnotetext{
* 10.29388/978-65-86678-46-8-0-f.181-192

${ }^{1}$ Personal Trainner. Graduado em Educação Física. Instrutor de Pilates e Neopilates. Instrutor de Técnica Respiratória Hipopressiva.

${ }^{2}$ Docente do Centro Universitário de Mineiros. Mestre em Saúde Coletiva. Especialista em Direito Civil Contemporâneo. Graduada em Educação Física e Direito.
} 
O método busca o equilíbrio físico e mental, aprimorando as capacidades físicas e corrigindo os desequilíbrios que cada indivíduo apresenta, por isso, é recomendado para praticamente todas as pessoas, independente de idade, sexo e profissão. O método é definido como uma junção entre ciência e arte, tendo sua linguagem própria, sendo ela clara e natural (APARICIO; PÉREZ,2005).

Ao longo do tempo, o método não conservou as bases iniciais propostas pelo seu criador Joseph Hubertus Pilates. Umas das justificativas para essa situação, foi a decisão proferida por um tribunal norte-americano que na ocasião julgou a respeito das características do método e a utilização do nome Pilates, tendo proferido decisão de classificar o método como genérico, dessa forma, houve a abertura de precedente para as pessoas utilizarem o nome para as mais diversas formas de aulas e para ganhos comerciais. Diante desse contexto as características iniciais do método e os princípios propostos pelo seu fundador passam por modificação e adaptações cotidianamente (APARICIO; PÉREZ,2005).

Segundo Aparicio e Pérez (2005) Joseph zelava pela forma de como seu método era repassado, com linguagem precisa, usando o idioma original, o inglês, assim criando seu próprio vocabulário. Instituições que utilizam o autêntico método Pilates, chamam atenção pelo vocabulário utilizado, onde é composto por frases de Joseph e de sua esposa Clara, em todo o mundo os professores usam esse mesmo vocabulário em seus respectivos idiomas.

O Método Pilates vem sendo amplamente difundido entre a população idosa que enfrenta as consequências do envelhecimento, que segundo Henriques e Farinatti (2013, p.3) “o envelhecimento é um fenômeno complexo que compreende alterações moleculares, celulares, fisiológicas e psicológicas”. Cruz (2013) ainda ressalta que o início da velhice ocorre, devido as questões fisiológicas, que são irreversíveis, no entanto, a depender do estilo de vida adotado, o ato de envelhecer passa a ser natural e não patológico, pois a qualidade de vida em todos os seus aspectos atua de maneira salutar nesse processo.

Segundo Henriques e Farinatti (2013, p.3)

O envelhecimento é um fenômeno complexo que compreende alterações moleculares, celulares, fisiológicas e psicológicas. Os problemas de saúde e o declínio fisiológico se desenvolvem progressivamente e, em parte, devem-se ao estilo de vida mantido ao longo dos anos. 
É descrito o crescimento da população idosa nas últimas décadas no mundo, o Brasil está entre os países onde esse aumento é significativo, quando comparado ao aumento da natalidade. Processo esse conhecido como transição demográfica e epidemiológica, que culminam em um envelhecimento populacional, invertendo a pirâmide etária nacional.

Esse panorama, traz um novo paradigma social, no sentido de buscar um envelhecimento saudável. É comum a preocupação com o processo de envelhecimento, e um dos maiores fatores de proteção atual para o não desenvolvimento de uma série de patologias, é a atividade física sistematizada, sendo uníssono na literatura científica especializada a importância da atividade física como ação principal na manutenção da longevidade (CRUZ, 2013).

Diante do exposto é importante o estudo da influência do Método Pilates nas pessoas idosas, uma vez que com o processo de transição demográfica e epidemiologia, a expectativa de vida no Brasil tem aumentado significativamente. Ademais o Método Pilates, apesar de não ser contemporâneo, ainda é pouco conhecido dos acadêmicos de educação física, que, na maioria das vezes conhecem apenas algumas características do método, através de notícias vinculadas na mídia por meio de telejornais, programas e rede sociais. Esse conhecimento de senso comum, não possui o condão de instrumentalizar uma prática profissional efetiva, sendo assim torna-se imprescindível o estudo do Método Pilates embasado nas suas bases científicas.

A partir disso, o objetivo do presente estudo foi compreender a influência do Método Pilates no processo de envelhecimento, tentando dessa forma, responder os seguintes questionamentos: o Método Pilates é indicado para toda população idosa?; quais os benefícios do Pilates nas capacidades físicas dos idosos?; a participação em aulas de Pilates influência os aspectos psicológicos dos idosos?

Para tanto foi realizada uma pesquisa a partir do método dedutivo, analisando os dados de maneira qualitativa, a partir de um levantamento bibliográfico realizado nas bases de dados Scielo e Lilacs, além da utilização de literatura pertinente ao tema, descrita em livros, principalmente foi utilizado como base para esse estudo no que concerne ao contexto histórico do método Pilates os autores Aparicio e Pérez (2005) e Isacowitz e Clippinger (2013). 


\section{Benefícios do método pilates}

Lima (2006) descreve o método Pilates como uma atividade física que proporciona vários benefícios aos adeptos, tais como, melhora do condicionamento físico, controle muscular, coordenação motora, ganhos de amplitude de movimento bem como de flexibilidade, melhora do aporte sanguíneo aos tecidos e orienta o corpo a uma postura adequada através da consciência corporal fornecida. Camarão (2004) cita ainda alívio das dores, aumento da força muscular e equilíbrio devido ao trabalho global que o método proporciona, assim garantindo uma vida mais saudável para os praticantes.

De acordo com Camarão (2004) os clientes do método procuram o Pilates com as seguintes queixas: má postura corporal, baixa resistência a exercícios físicos, dores coluna e grandes encurtamentos musculares. $\mathrm{O}$ Pilates tem se efetivado como um ótimo sistema preventivo, que melhora o alongamento geral, estabiliza as curvas fisiológicas da coluna, estrutura a postura, evitando deformidades patológicas tais como cifoses e escolioses. Santos e Barreto (2001) explicam que um bom controle postural beneficia o praticante como proteção contra traumas nas estruturas do corpo e melhora a funcionalidade. Gómez e Garcia (2009), asseguram que o Pilates em relação a reeducação postural, é muito eficaz.

A flexibilidade entre outros benefícios do método apresenta uma correlação benéfica com a reeducação postural (KOLYNIAK et al. 2004). Quando o corpo apresenta uma boa flexibilidade, a incidência de lesões é diminuída, porém é importante ressaltar que está em excesso poderá causar instabilidade articular (BERTOLA et al. 2007).

O Pilates envolve todos os tipos de contração muscular, principalmente a isométrica com ênfase no "powerhouse", a qual promove a estabilização estática e dinâmica. Conforme os princípios de concentração, controle motor e precisão dos exercícios, abordados pelo método, estes parecem intensificar o alinhamento e a percepção corporal, através das respostas proprioceptivas (MARES et al. 2012).

Contreras, (2010) enaltece a importância do sistema proprioceptivo dando ênfase no equilíbrio, pois o cérebro mantém em funcionando o que é de fato estimulado. No método Pilates são utilizados acessórios, tais como; rolos, bolas, meia-lua e exercícios de instabilidade para promover o estímulo do equilíbrio, sendo assim, o cérebro registra a forma de equilíbrio utilizada e consegue manter esse benefício ao corpo dos adeptos a modalidade. Muitas pessoas não conseguem realizar determinadas atividades, não por falta de força ou resistência, mas, por falta de 
coordenação e equilíbrio, qualidades que muitas vezes não são suficientemente trabalhadas por algumas atividades físicas.

Contreras (2010) acrescenta que o método Pilates é uma maneira de estimular essas qualidades seguindo dois princípios básicos sendo eles como a concentração e o controle, dessa forma, estimulam um grande número de informações enviadas ao cérebro promovendo a consciência e harmonia dos movimentos. Segundo Joseph Pilates, a cartela de benefícios para os praticantes do método Pilates está diretamente ligada a associação de uma boa execução dos exercícios com a integridade dos seus princípios (CAMARÃO, 2004).

\section{Pilates e o idoso}

Dentre as inúmeras modalidades de atividade física existente que pode ser trabalhada com o idoso, o método Pilates vem ganhando visibilidade e sendo bastante indicado para esse público, além de ser fortemente aceito pelos praticantes desta faixa etária. Estudos de revisão sistemática enaltecem a atuação positiva do Pilates para a saúde de idosos e aborda ganhos positivos na composição corporal, aumento de força e resistência muscular, melhora do equilíbrio, coordenação, flexibilidade, respiração e postura corporal (CANCELA et al 2014).

A partir de todos os benefícios biológicos, a prática do Pilates consequentemente influência a qualidade de vida dos idosos. Estudo realizado por Costa et al. (2018), verificou a percepção da qualidade de vida em idosas praticantes e não praticantes de Pilates, através do questionário WHOQOL-OLD, encontrando como resultados maiores escores na maioria dos domínios da qualidade de vida na faixa etária compreendida entre 70 a 74,9 anos.

Da mesma forma Mello et al. (2018) estudaram a qualidade de vida e a satisfação com a saúde a partir da prática do método Pilates, relatando que houve um aumento significativo na consideração da qualidade de vida, que antes da intervenção era considerada como muito boa por 30,7\% dos idosos, e após intervenção esse percentual aumentou para 92,3 \%. O estudo apresentou ainda melhoria da satisfação em relação à saúde de $23,0 \%$ para $30,7 \%$ e a disposição para a realização de tarefas cotidianas de $7,6 \%$ para $30,7 \%$.

Esses dados devem ser analisados com parcimônia, vez que, a amostra desse estudo era muito pequena, o que dificulta possíveis extrapolações, no entanto, por se tratar de um estudo de campo, os resultados 
podem sugerir que se acaso fosse utilizado uma amostra maior os resultados provavelmente se repetiriam.

A qualidade de vida é influenciada por diversos aspectos, além de certa forma ser uma categoria que é permeada por uma certa subjetividade. Aspectos sociais, econômicos, psicológicos, emocionais e biológicos influenciam a qualidade de vida ou mesmo a percepção da mesma por parte do indivíduo. Dentre esses fatores, a prática do exercício físico tem se mostrado essencial em contribuir para uma qualidade de vida satisfatória.

Ocorre que, o indivíduo para praticar algum tipo de atividade física sistematizada precisar iniciar e continuar na mesma, e muitas vezes, apesar de começar tão logo desiste, pois falta um requisito básico para a aderência a uma vida mais ativa, que é a motivação para realizar tal prática. É comum na população idosa essa motivação ser relacionada a saúde, diferentemente do público adulto que em sua maioria na sociedade contemporânea busca quase que exclusivamente a estética como a principal forma de motivação.

Diante do exposto, Dantas et al. (2015) avaliaram os fatores motivacionais de idosos que praticavam o método Pilates, a partir do Inventário de Motivação à Prática Regular de Atividades Físicas (IMPRAF$54)^{3}$. Nesse estudo, a saúde foi o maior fator motivacional apontados pelos idosos, em sequência a sociabilidade e o prazer. Essa pesquisa apresentou os dados em comparação ao público masculino e feminino, sendo que no caso da dimensão sociabilidade o público masculino apresentou motivação alta em relação a essa dimensão.

Essa pesquisa trouxe um dado importante, e que merece ser estudado em futuras pesquisas de campo, quando o objeto de estudo for o método Pilates e as variáveis relacionadas ao exercício físico, qualidade de vida, saúde entre outros, que é a participação do sexo masculino. Os estudos científicos na temática do Pilates ainda apresentam uma baixa prevalência quando comparado a outras práticas físicas, e principalmente o público idoso aparece muito pouco nas pesquisas (CARVALHO, et al. 2017). É plausível supor que isso ocorra pelo fato de que a maioria dos praticantes dessa prática corporal sejam mulheres, no entanto, essa situação deve ser melhor estudada para futuras conclusões. Carvalho et al. (2017, p. 232) discutem essa limitação:

\footnotetext{
${ }^{3}$ Invetário IMPRAF- 54- é composto por um questionário com 54 itens dividido em nove blocos, que são subdivididos em seis domínios a saber: controle de estresse, saúde, sociabilidade, competitividade, estética e prazer.
} 
Outra limitação observada através da pesquisa é que a maior parte da amostra dos estudos trabalhados encontrados era composta pelo público feminino, ou em alguns casos, públicos mistos, o que indica a maior adesão de mulheres idosas a prática de Pilates e impossibilita a extrapolação para o público masculino devido às particularidades do sexo.

Em relação a população idosa, quando se pretende avaliar a independência funcional, uma categoria amplamente difundida é a capacidade funcional, que considera a interação entre a pessoa e o seu ambiente cotidiano. A capacidade funcional pode ser definida como atributos intrínsecos, ou relacionados à saúde que permite que um indivíduo consiga realizar as atividades básicas e instrumentais da vida diária (OMS, 2015).

Nesse sentido, o Pilates e seus efeitos na autonomia funcional de idosas foi estudado por Rodrigues et al. (2010) que selecionaram 52 idosas que participaram de uma avaliação geral ${ }^{4}$ e do protocolo de avaliação da autonomia funcional do Grupo Latino-americano de Desenvolvimento para a Maturidade (GDLAM) ${ }^{5}$. Esse estudo foi desenvolvido a partir do grupo praticante de Pilates (GP) e grupo controle (GC).

O GP apresentou resultados estatisticamente melhores em todos os testes e no índice geral do GDLAM, após a intervenção de uma série de 10 exercícios de Pilates, por pelo menos 2 duas vezes por semana, com duração de 1 hora por sessão. O GC apenas apresentou escore significativamente melhor no teste de caminhada de 10 metros.

Carvalho et al. (2017) analisaram a partir de uma revisão sistemática a capacidade funcional do idoso e a influência o método Pilates, e concluíram que o referido método promoveu melhoras significativas na autonomia funcional dos idosos. No entanto, relataram no estudo que encontraram um número muito pequeno de estudos que avaliaram a capacidade funcional, e que a maioria dos estudos que tratam do método Pilates avaliaram características isoladas e não propriamente a capacidade funcional, através de instrumentos específicos.

Apesar da capacidade funcional ser o maior parâmetro de estudos e análises na população idosa, a aptidão física também deve ser avaliada, principalmente os componentes relacionados a saúde. A aptidão física é dividida na literatura de maneira didática, relacionada a saúde e ao

\footnotetext{
${ }^{4}$ Avaliação geral mensurados peso e idade e da autonomia funcional.

${ }^{5}$ Esse protocolo consiste em testes de caminhada de 10 metros, levantar-se da posição sentada, levantar-se da cadeira e locomover-se pela casa, levantar-se da posição de decúbito ventral e vestir e tirar uma camiseta.
} 
desempenho. Dessa forma, as capacidades físicas de resistência aeróbica, força, resistência muscular localizada, flexibilidade, além da composição corporal são relacionadas a saúde; e as capacidades físicas como a agilidade, velocidade, potência, capacidade anaeróbica e equilíbrio são comumente relacionadas ao desempenho.

Embora a partir dessa divisão didática, quando se trata da população idosa a capacidade física de equilíbrio é essencial para a manter e aprimorar a saúde e independência para as ABVD (atividades básicas da vida diária) e AIVD (atividades instrumentais da vida diária). Dessa forma, estudo realizado por Rodrigues et. al, (2009), avaliaram o equilíbrio estático em idosas após treinamento com o método Pilates, a partir do protocolo de Tinetti $^{6}$.

Esse estudo a exemplo do estudo citado anteriormente também utilizou dois grupos, classificados como GP e GC. O GP realizou os exercícios durante oito semanas com frequência de duas vezes semanais e sessões de 60 minutos, sendo que o GC não sofreu nenhum tipo de intervenção. Os resultados mostraram que o GP obteve diferenças estatisticamente significativas no equilíbrio estático, concluindo dessa maneira que as idosas submetidas à prática do Pilates possuíam um melhor equilíbrio estático quando comparadas as idosas que não praticavam o Pilates.

O equilíbrio foi descrito no estudo realizado por Costa et al. (2016), como uma variável que é aprimorada a partir do treinamento do método Pilates, além do risco de queda diminuído, e a flexibilidade aumentada. Esse estudo foi realizado a partir de uma revisão integrativa ${ }^{7}$ que incluiu estudos com idosos submetidos à intervenção com o método Pilates, e que a partir dos critérios de seleção foram analisados 17 artigos em uma população de 445 estudos.

Os autores citam ainda que em outras variáveis relatadas na literatura acerca da influência do treinamento do método Pilates, ainda existem dados controversos e sugerem que: "[...] a realização de ensaios clínicos, garantindo maior confiabilidade dos efeitos do Pilates, possibilitando a concatenação dos resultados em futuras revisões sistemáticas com metanálise" (COSTA et al. 2016, p. 701).

Mello et al. (2018) também realizaram um estudo com 13 idosos a fim de verificar os efeitos do Método Pilates, na aptidão física, corro-

\footnotetext{
${ }^{6}$ Protocolo que avalia o equilíbrio estático, dividido em equilíbrio orientado pela performance e a da marcha orientada pela performance.

${ }^{7}$ Revisão que utiliza a busca, avaliação e sistematização das evidências científicas exis tentes, a partir das bases de dados MEDLINE ( PubMed), Scopus e Physiotherapy Evidense Database (PEDro).
} 
borando com os resultados do estudo de Costa et al. (2016) no que diz respeito a variável flexibilidade, o que é comum esperar de estudos relacionados ao Pilates, pois essa prática é realizada a partir de muitos exercícios de alongamento o que culmina na melhora da flexibilidade. No estudo de Mello et al. (2018) também houve diferenças significativas entre o pré e o pós- testes nas variáveis de agilidade, resistência aeróbica e equilíbrio dinâmica, aumentando dessa forma a aptidão física dos participantes.

O centro de força corporal conhecido na área da Educação Física por região do core e no Método Pilates como powerhouse, é uma estrutura vital do desenvolvimento das capacidades físicas e influencia diretamente a capacidade funcional. Aparicio e Pérez (2005, p. 37) discorrem sobre o centro de força:

O centro de força é definido como o "cinturão" (anterior e posteriormente falando) que se estende desde a base das costelas até a região inferior da pélvis [...]. O centro suporta o tronco, ajuda a melhorar a postura, facilita movimentos equilibrados e afina $o$ controle motor das extremidades[...]. O desenvolvimento do centro de força ajuda a prevenir a dor nas costas e outras lesões, e implica menos fadiga.

Essa estabilidade central do corpo foi estudada no método Pilates a partir de uma revisão sistemática de literatura proposta por Marés et al. (2012), na qual os autores concluíram que embora sejam necessários mais estudos sobre a temática, os estudos da revisão em tela demostraram que a estabilização central é primordial no método Pilates, pois a partir desta há um equilíbrio efetivo de carga na coluna vertebral pélvis e cadeia cinética.

Embora esse estudo não tenha referência específica à população idosa, esses achados são interessantes, pois se eles são importantes para as populações em geral, não deixariam de o ser para as populações idosas, principalmente no que diz respeito a coluna vertebral, que no processo de envelhecimento passa por processos deletérios, e a depender das atividades básicas e instrumentais realizadas ao longo da vida, pode apresentar diversos desvios como escoliose, hiperlordose, cifose, protrusão de vértebras, hérnias de disco, entre outras.

Em se tratando de distúrbios da coluna lombar Kolyniak et al. (2004), relataram que o desequilíbrio entre a função dos músculos extensores e flexores de tronco é um potente indicador para a apresentação 
dos mesmos. Estudaram a partir do método Pilates a função dessa musculatura. Concluíram que o método Pilates é uma prática corporal eficiente no fortalecimento da musculatura extensora do tronco, podendo diminuir o desequilíbrio entre a função dos músculos implicados na flexão e extensão do tronco.

É importante destacar que esse estudo está imbuído de particularidades, como o nível de treinamento dos participantes da pesquisa, que no caso apresentado, praticaram os exercícios do método Pilates durante 12 semanas no nível intermediário a avançado. Ocorre que, não necessariamente a população idosa praticante de Pilates conseguirá alcançar esse nível de treinamento, isso dependerá de diversas variáveis como o tempo de prática, inicio do treinamento, propriocepção corporal dentre outras características, no entanto, ainda assim são resultados que devem ser considerados, sendo que seria importante a replicação de tal estudo na população idosa para comparação dos resultados.

\section{Considerações finais}

O presente estudo confirma a eficácia do Método Pilates quando aplicado a população idosa, pois este evidencia grande melhora nas atividades de vida diária, o que garante independência, bem como, traz benefícios em diversos domínios, como o biológico, psicológico e social. Sendo assim o Método Pilates é uma ferramenta de potencial para melhorar e conservar a saúde, bem como, prevenir as enfermidades que surgem no decorrer do envelhecimento, pois proporciona aos praticantes ganho de força muscular, resistência, flexibilidade, estabilidade postural, melhora na respiração e na postura corporal.

\section{Referências}

APARICIO, Esperanza.; PÉREZ, Javier. O autêntico método Pilates: a arte do controle. São Paulo: Planeta do Brasil, 2005.

BERTOLLA, Flavia et al.; Efeito de um programa de treinamento utilizando o método Pilates na flexibilidade de atletas juvenis de futsal. Revista Brasileira de Medicina do Esporte. v.13, n.4, 2007.

CAMARÃO, Teresa. Pilates no Brasil: corpo e movimento. Rio de Janeiro: Elsevier, 2004.

CANCELA, Jose M. OLIVEIRA, Iris M.; RODRIGUEZ-FUENTES, Gustavo. Effects of Pilates method in physical fitness on older adults: a systematic re- 
view. European Review of Aging and Physical Activity. v. 11, n. 2, p. 81-94, 2014.

CARVALHO, Cinara Brandão de Oliveira et al.; O método Pilates e sua influência na capacidade funcional do idoso: uma revisão sistemática. Revista Kairos-Gerontologia. São Paulo, v.20, n.3, p. 223-235, 2017.

CONTRERAS, Ruth Espinosa. Como desenvolver a coordenação motora e o equilíbrio. Revista Oficial de Pilates. v.1, n. 04, Editora Online, 2010.

COSTA, Letícia Miranda Resende et al.; Os Efeitos do Método Pilates Aplicado à População Idosa: Uma Revisão Integrativa. Revista Brasileira Geriatria Gerontologia. Rio de Janeiro, v.19, n.4, p. 695-702, 2016.

COSTA, Tiago Rocha Alves et al.; Comparação da percepção da qualidade de vida em idosas praticantes e não praticantes do Método Pilates. Cadernos Saúde Coletiva. Rio de Janeiro, v.26, n.3, p.261-269, 2018.

CRUZ, Francine. Educação Física na Terceira Idade: Teoria e Prática. 1.ed. São Paulo: Ícone, 2013.

DANTAS, Ricardo et al.; Fatores Motivacionais De Idosos Praticantes Do Método Pilates. Revista Brasileira Promoção Saúde. Fortaleza, v.28, n.20, p. 251-256, 2015.

GÓMEZ, Virginia Serrano. GARCÍA, Oscar García. Ejercicio físico y Pilates durante el embarazo. Revista Digital. Buenos Aires, v.14, n. 136, 2009.

HENRIQUES, Patrícia.; FARINATTI, Paulo de Tarso Veras. Envelhecimento, nutrição e exercício. In: Envelhecimento, promoção da saúde e exercício: tópicos especiais em aspectos biológicos e psicossociais. v.2. Barueri, São Paulo: Manole, 2013

ISACOWITZ, Rael.; CLIPPINGER, Karen. Anatomia do Pilates. Barueri, São Paulo: Manole, 2013.

KOLYNIAK, Ester Garcia Garcia et al.; Avaliação isocinética da musculatura envolvida na flexão e extensão do tronco: efeito do método Pilates. Revista brasileira de medicina do esporte. Niterói, v. 10, n. 6, 2004.

LIMA, Ana Paula Peixoto. Os efeitos do método pilates em mulheres na faixa etária de 25 a 30 anos com lombalgia crônica. Cuiabá 2006, (monografia) Trabalho de Conclusão de Curso; Universidade Federal de Mato Grosso. Faculdade de Educação Física, do Campus de Cuiabá; ago. 2006.

MARES, Gisele Oliveira et al.; A importância da estabilização central no método Pilates: uma revisão sistemática. Fisioterapia Movimento. v. 25, n. 2, p. 51 445, 2012. 
MELLO, Natalia Ferraz et al.; Método Pilates Contemporâneo na aptidão física, cognição e promoção da qualidade de vida em idosos. Revista Brasileira Geriatria Gerontologia. Rio de Janeiro, v.21, n.5, p. 620-626, 2018.

OMS. Organização Mundial da Saúde. Relatório Mundial de Envelhecimento e saúde. 2015. Disponível em: < https://sbgg.org.br/wp-content/uploads/ 2015/10/OMS-ENVELHECIMENTO-2015-port.pdf $>$.

RODRIGUES, Brena Guedes de Siqueira et al.; Autonomia funcional de idosas praticantes de Pilates. Fisioterapia e Pesquisa, São Paulo, v.17, n.4, p.3005,2010 .

RODRIGUES, Brena Guedes de Siqueira et al.; Avaliação do equilíbrio estático de idosas pós- treinamento com método pilates. Revista Brasileira Científica e Movimento. v.17, n.4, p. 25-33,2009

SANTOS FILHO, Serafim Barbosa. BARRETO, Sandhi Maria. Atividade ocupacional e prevalência de dor osteomuscular em cirurgiões dentistas de Belo Horizonte, Minas Gerais, Brasil: contribuição ao debate sobre os distúrbios osteomusculares relacionados ao trabalho. Caderno Saúde Pública. v.17, n.1, p.181-93, 2001. 